\title{
Effects of Field of View on Balance in an Immersive Environment
}

\author{
Henry B. L. Duh ${ }^{1}$, James J.W. Lin ${ }^{1}$, Robert V. Kenyon ${ }^{2}$, Donald E. Parker ${ }^{3}$, Thomas A. Furness ${ }^{1}$ \\ Human Interface Technology Lab \\ University of Washington \\ Seattle, $W A^{l}$ \\ Department of Electronic Engineering and \\ Computer Science \\ Department of Otolaryngology \\ University of Illinois at Chicago \\ University of Washington \\ Chicago, $I L^{2}$ \\ Seattle, $W A^{3}$
}

\{duhbl, jwlin, kenyon, deparker, tfurness\} @ hitl.washington.edu

\begin{abstract}
With technological progress, wide field-of-view (FOV) displays will become increasingly common. Wide FOVs provide a more immersive environment and produce stronger self-motion perception. The objective of this study was to investigate the relationships between FOV and scene content on postural stability in an immersive environment. 10 subjects were tested using two different scenes (a simple radial pattern and a "meaningful" city scene) at six FOVs $\left(30^{\circ}, 60^{\circ}, 90^{\circ}\right.$, $\left.120^{\circ}, 150^{\circ}, 180^{\circ}\right)$ using a within-subjects design. Subjects exhibited more postural disturbance with increasing FOV. A surprisingly large increase in disturbance was found for the interval between $150^{\circ}$ and $180^{\circ}$ using the city scene. No statistically significant difference was found for effects of scene content. Two groups (postural stable group and postural unstable group) were identified during experiment. These groups performed differently in the two scene conditions. Future research plans are described in the Discussion.
\end{abstract}

\section{Introduction}

Vection, visually-induced self-motion, may contribute both to 'presence' in virtual environments and to simulator sickness (SS) [14]. People usually report higher incidence of SS with a wide than a narrow FOV display [15]. A wide FOV display can maximize immersion of the user in the virtual environment; a limited FOV may degrade the sense of presence [20].
Determining the region of the retina most responsible for the self-motion perception has been pursued by several investigators [see 22]. Researchers initially reported that stimulation of peripheral areas of the retina was more effective in eliciting self-motion perception than simulation of more central areas [4], [8].

It has been theorized that human vision is associated with two functionally different systems. Leibowitz and Post [18] extended the notion of "two modes of processing spatially distributed information" which was proposed by Held [7] and others. The two-modes model described two different kinds of visual functions mediated by different brain regions. These are the focal mode and the ambient mode. The former was thought to be responsible for object recognition and identification and concerned with the 'what' question. The later was thought to be responsible for spatial orientation, locomotion and posture and concerned with the 'where' question.

Several studies examined this theory. Brandt, Dichgans, and Koenig [2] reported that when the central retina was stimulated, self-motion was not experienced, but strong self-rotation was elicited when the peripheral retina was exposed to optical flow. Hulk and Rempt [11] using sine-wave grating of various widths, found that self-motion was most frequently reported at FOV eccentricities of $50^{\circ}$ and $60^{\circ}$ with the slower angular velocities $\left(10^{\circ} / \mathrm{sec}-15^{\circ} / \mathrm{sec}\right)$ proving most effective. Howard and Heckmann [10] reported that when stimuli were presented in the peripheral visual field, self-motion was stronger than when the stimuli were presented in the central field. However, 
vection was reduced when the central stimuli moved opposite to the direction of the peripheral stimuli.

DiZio and Lackner [5] evaluated 21 subjects in two different FOV conditions. Subjects reported more motion sickness symptoms when using the wide FOV head-mounted display ( $138^{\circ}$ horizontal by $110^{\circ}$ vertical $)$ than did those who were exposed to a FOV half as large. Kenyon and Kneller ??? [16] examined a visual nulling task at five different FOVs $\left(10^{\circ}, 20^{\circ}, 40^{\circ}, 80^{\circ}\right.$ and $120^{\circ}$ ). They found that subjects performed best in the $80^{\circ}$ FOV condition, not at $120^{\circ}$, as expected. Subjects also reported greater task difficulty at $120^{\circ}$ FOV. Kenyon and Kneller suggested that subjects experienced stronger vection at the $120^{\circ} \mathrm{FOV}$, which made the task more difficult.

These studies suggest that the experience of selfmotion depends in part on motion cues in the peripheral region of the retina. Wide FOV displays allow more immersion in the virtual environment which may enhance the experience of 'presence'. On the other hand, stronger vection, which is one of the factors that may contribute to SS, is also likely be reported. The objective of this study was to investigate

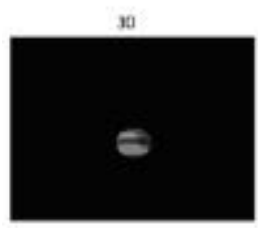

100

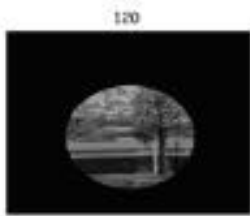

30

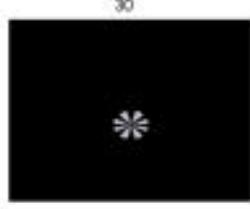

120

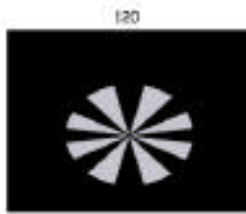

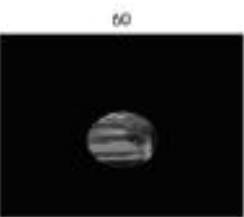

150

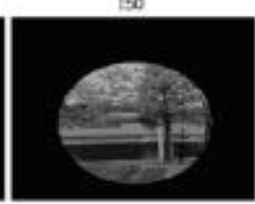

60

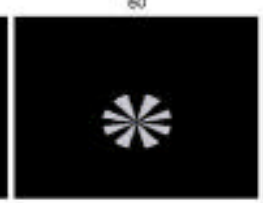

100

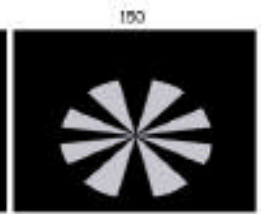

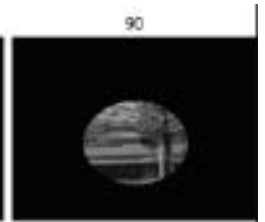

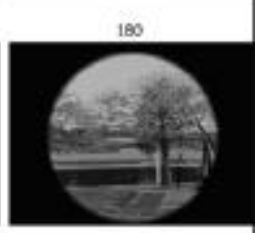

x

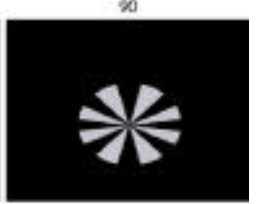

190

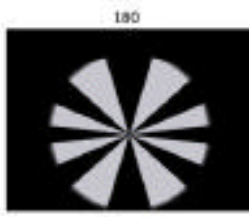

Figure 1. Visual scenes. The upper panels show the Edmonds city scene. The lower panels illustrate the simple radial pattern. effects of different FOVs and different scene contents on postural stability in an immersive environment.

\section{Experiment}

\section{Method}

Subjects. 5 women and 5 men, ages 20 to 30, were recruited from the Human Interface Technology Laboratory subject pool. None reported a history of auditory disturbance, balance disorders, back problems, or high susceptibility to motion sickness. All subjects reported that they had normal or corrected vision. Subjects were paid $\$ 10 /$ hour. The protocol was approved by the University of Washington Human Subjects Review Committee.

Apparatus. Visual scene motion was generated by computer software. This software accesses a series of digitized images to simulate scene motion. Two computer-generated scenes (city scene - Edmonds, WA and a simple scene - radial pattern) were used (see Figure 1). The images projected by a Box-Light projector (Box-Light, Inc.) were presented on a 3-foot dome, which has a nominal $180^{\circ} \mathrm{x} 180^{\circ} \mathrm{FOV}$, at a 640 $\mathrm{X} 480$ pixel resolution. Subjects stood on a Chattecx balance platform (Chattecx Corp.) that automatically calculated dispersion around the center-of-balance based on signals generated by force plates under their feet. Dispersion is calculated by determining mean center-of-balance (COB) along $\mathrm{X}$ and $\mathrm{Y}$ axes. The squared deviations of sampled points from the COBs are used to calculate a standard deviation - the dispersion index. The experiment setting is illustrated in Figure 2.

Procedure. Frontal visual scene roll oscillation was presented at a low frequency - $0.05 \mathrm{~Hz}$ (see Parker, Duh, Philips and Furness, [19]). Peak scene angular

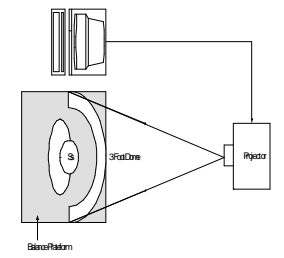

Figure 2. Equipment layout 
Standardized Dispersion

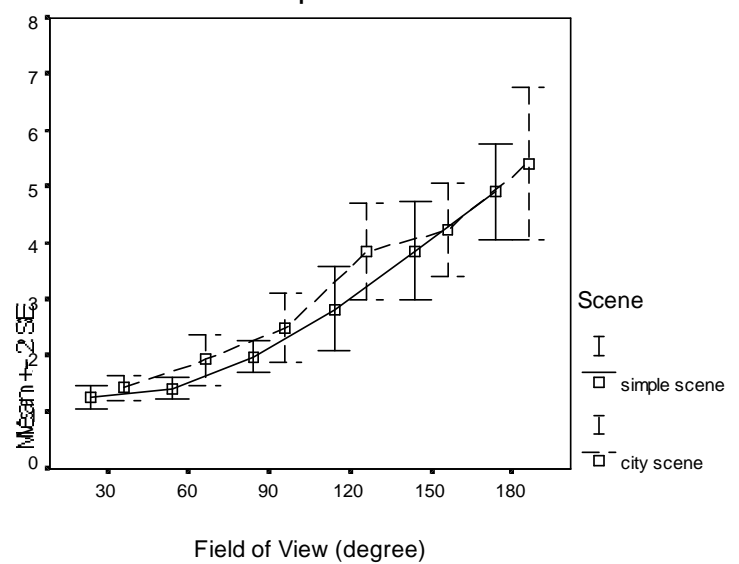

Standardized Rating

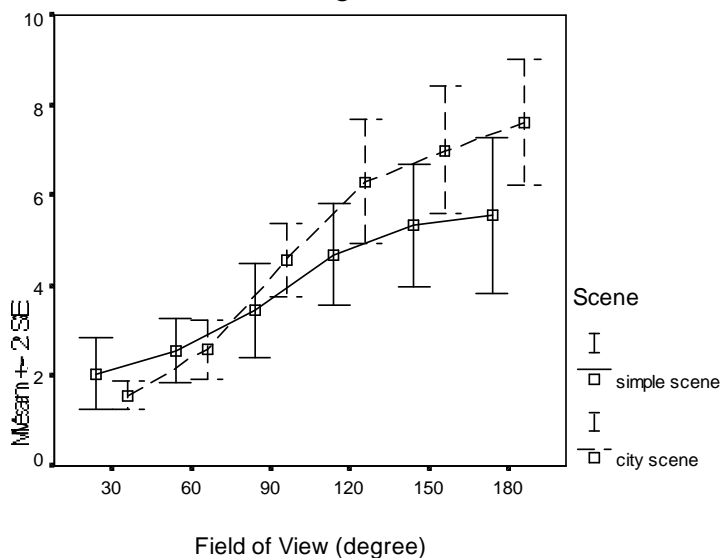

Figure 2. Standardized rating and dispersions as a function of field of view for simple and city scene - means and standard errors. (Note: to preserve readability of the error bars, mean data points for the FOV conditions are staggered.)

velocity was constant at approximately $70^{\circ} / \mathrm{sec}$. Scene update rate was approximately 40 frames/sec. Scenes were presented at 6 different of FOVs $\left(+/-15^{\circ},+/-30^{\circ}\right.$, $+/-45^{\circ},+/-60^{\circ},+/-75^{\circ},+/-90^{\circ}$ from the center of the visual field). Data were collected with the subjects in a sharpened Rhomberg stance; i.e., subjects stood on the balance platform with one foot in front the other and with their arms crossed behind their backs, as described by Parker, Duh, Philips and Furness [19]. The balance system collects data at a sampling rate of $100 \mathrm{~Hz} .4$ trials (replicates) were collected in each stimulus condition. 10-sec periods of baseline data while viewing a static scene were collected before and after the moving scene trials. For the latter, the subjects looked at the moving scene for $10 \mathrm{sec}$ while holding the support bars, assumed the Rhomberg position, and attempted to stand steady during the $10 \mathrm{sec}$ data collection. The subjects also attempted to estimate the difficulty they experienced in maintaining their balance. Subjects' eyes were closed except during the visual stimulus trials. We determined all possible orders of experimental conditions and randomly choose a different order of FOV conditions for each subject. All subjects finished the simple scene condition first, then came back to finish the city scene condition one week later.

The following data were collected for each trial: stance break (yes, no); latency to stance break (10 sec maximum); subjective difficulty rating (1-10 scale); dispersion of center-of-balance. A stance break occurred when subjects uncrossed their arms or moved their feet off the force plates.

\section{Results}

Results from this study are summarized in Figure 2. Subjects exhibited increased center-of-balance dispersion with increasing FOV for both the city and simple scenes. For the simple scene, there was a statistically significant main effect of FOV calculated using a repeated measures analysis of variance (ANOVA) $[\mathrm{F}(5,5)=9.367, \mathrm{p}<0.05]$; for the city scene, the FOV main effect was also significant $[F(5,5)=7.72, p<0.05]$. There was a significant main effect of FOV for the rating data in the city scene condition $[F(5,5)=9.801, \mathrm{p}<0.05]$. Post hoc analysis of the simple scene data indicated that, except for the $30^{\circ}-60^{\circ}$ interval, all the intervals were significantly different. The largest difference was for the $120^{\circ}-150^{\circ}$ interval. For the city scene data, the $60^{\circ}-90^{\circ}, 90^{\circ}-120^{\circ}$ and $150^{\circ}-180^{\circ}$ intervals were significantly different; the largest difference was for the $90^{\circ}-120^{\circ}$ interval.

\section{Discussion}

All the data showed the same trend - with increasing FOV, subjects exhibited more dispersion and reported more difficulty keeping their balance. For the city scene data, the dispersion increase for the $150^{\circ}-180^{\circ}$ interval was nearly as large as for the $90^{\circ}-120^{\circ}$ interval. Failure to observe 'saturation' at the extreme FOVs was surprising.

With increasing FOV, subjects received more information from their peripheral visual field, which 


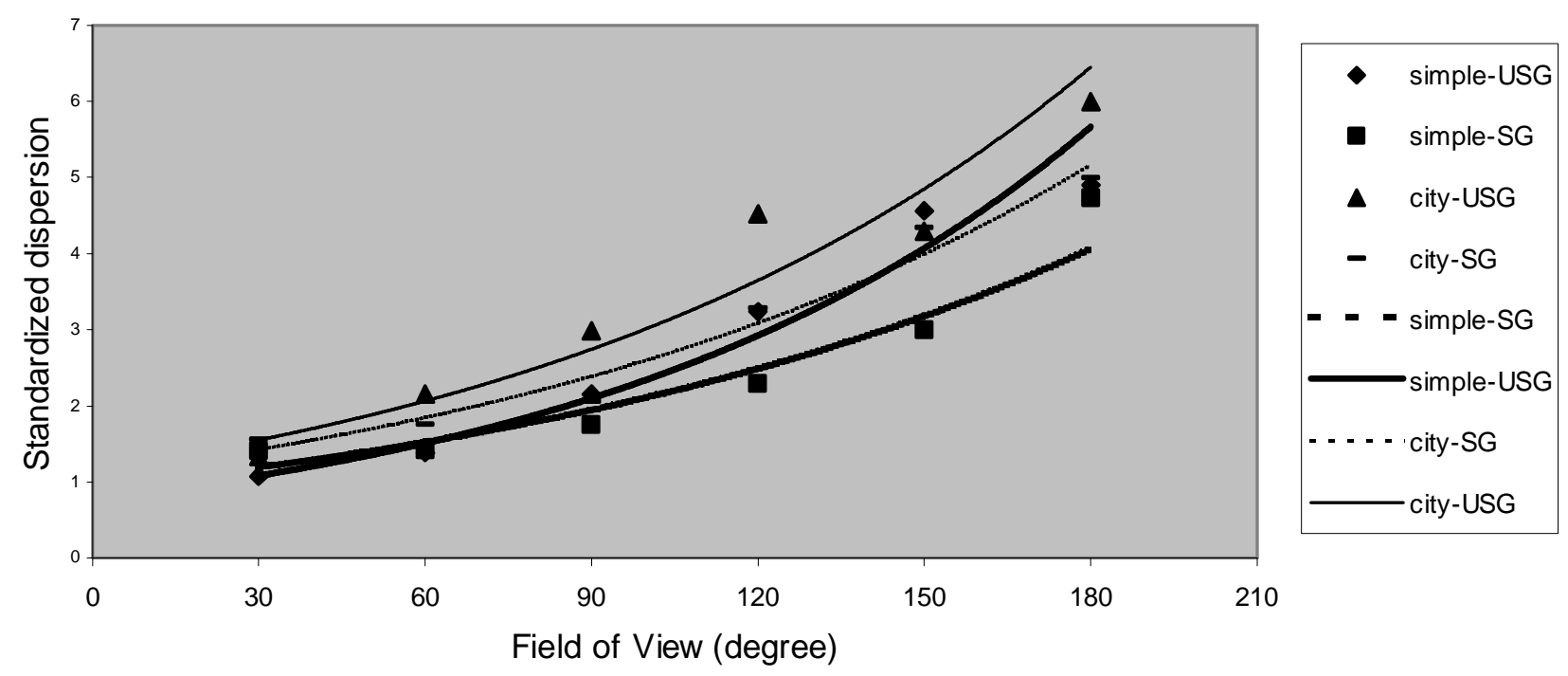

Figure 3. Dispersion as a function of FOV - best-fitting exponential curves for the SG and the USG in two scene conditions.

apparently caused greater postural disturbance. These findings support the assertion that wide FOVs cause greater self-motion perception. Regarding the scene conditions, it seems that different scenes had different effects on postural stability. Subjects exhibited more dispersion and more balance difficulty with the city scene than with the simple scene. However, there were no statistically significant differences between the simple and city scene conditions for either the standardized dispersion or rating data. Keshner and Kenyon [17] found no effect of scene complexity in their study of posture using an architecture scene and a field of random dots.

For some subjects, postural disturbance appeared to saturate by the $150^{\circ}$ FOV. Based on individual dispersion data at $150^{\circ}$, we separated subjects into two groups - group1 was a stable group (SG, dispersion below the median), group2 was the less stable group (USG, dispersion above the median). If we plot the data as exponential curves, as shown in Figure 3, it is clear that the city scene evoked greater postural disturbance than the simple scene. The shapes of the curves for both the SG and USG groups are similar. The curves differ primarily in their lateral position along the FOV axis. In our experiment, the most significant difference between FOV intervals occurred between $120^{\circ}-150^{\circ}$ for the simple scene. However, with the city scene the most significant difference occurred between $90^{\circ}-120^{\circ}$. It is possible that if we presented different scene content, subjects would behave differently.

Each exponential curve can be fit to a regression model. For simple/USG, $\mathrm{y}=0.010 \mathrm{x}+0.785, \mathrm{R}^{2}=0.825$ (here $\mathrm{y}=\ln [$ standardized dispersion], $\mathrm{x}=\mathrm{FOV}$ ); city/USG: $\quad \mathrm{y}=0.009 \mathrm{x}+1.115, \quad \mathrm{R}^{2}=0.630 ; \quad$ simple/SG: $\mathrm{y}=0.008 \mathrm{x}+0.920, \quad \mathrm{R}^{2}=0.716 ; \quad$ for $\quad$ city $/ \mathrm{SG}$, $y=0.008 x+1.049, R^{2}=0.689$. (See Table 1.) As can be seen, the coefficients for different scenes are similar but the intercepts are slightly different. For both slope and intercept regression coefficients, we examined mean differences between the city the simple-radial scene conditions for the unstable group. Similar calculations were performed for the stable group. There were no statistically significant effects for either slope or intercept for simple/USG - city/USG (slope: $\mathrm{t}=-1.124, \mathrm{p}=0.266$; intercept: $\mathrm{t}=-1.797, \mathrm{p}=0.078$ ) and for simple/SG - city/SG (slope: $\mathrm{t}=0.43, \mathrm{p}=0.669$; intercept: $\mathrm{t}=-0.693, \mathrm{p}=0.491$ ). This suggests that both groups behaved similarly across scene conditions. There was also no statistically significant difference for city/USG - city/SG (slope: $\mathrm{t}=-0.275, \mathrm{p}=0.784$; intercept: $\mathrm{t}=0.766, \mathrm{p}=0.447$ ); but, for simple/USG simple/SG there were significant differences for both slopes and intercepts (slope: $\mathrm{t}=-2.169, \mathrm{p}=0.034$; intercept: $\mathrm{t}=-2.166, \mathrm{p}=0.037)$. This means that the $\mathrm{SG}$ and the USG behaved differently for simple scene but not for the city scene. 
It is interesting that the groups performed differently in simple scene but not in city scene. It is possible that city scene provided more detailed horizontal and vertical cues than the simple scene. When the scene is more compelling (in our experiment, the city scene caused more balance disturbance), the effects of content across groups decreased. These results may be related to so-called visual field dependent / visual field independent perceptual styles. Barrett, Thornton and Cabe [1] examined the relationship between perceptual style and cue conflict. They found that field-dependent people experienced the most discomfort. Isableu et al. [12] investigated the relationship between the perceptual style and postural control. They found that field-dependent people were less stable than fieldindependent people. The field-dependent people required dynamic visual cues to maintain their postural stability. In our experiment, USG group exhibited larger balance disturbance than SG group in the simple scene condition. USG people may be more reliant on visual cues to maintain their balance. Individual differences in perceptual style differences may be important determinants of responses to scene content.

Why was the most significant FOV difference for the simple scene between $120^{\circ}$ and $150^{\circ}$ whereas the most significant difference in the city scene was between $90^{\circ}$ and $120^{\circ}$ ? We suggest two possibilities. First, each eye has an individual FOV of $150^{\circ}$ horizontally. The overlap region (binocular FOV) in the center averages $120^{\circ}$ with $30^{\circ}-35^{\circ}$ monocular vision on each side. The combined horizontal FOV is $180^{\circ}$. The ideal display should have a total horizontal FOV of $180^{\circ}$, each eye having a $150^{\circ} \mathrm{FOV}$, with binocular overlap of $120^{\circ}$ FOV. The city scene provided more complex visual information and more meaningful objects. Subjects appeared to be more involved in this scene. Second, when we increased the FOV, subjects exhibited saturation at lower FOVs for the city scene than for the simple scene. As suggested previously, the city scene had more horizontal and, vertical cues and more meaningful objects. This suggests the following question: would a higher resolution scene cause more disturbance than a lower resolution scene?

\begin{tabular}{|l|l|c|}
\hline & \multicolumn{1}{|c|}{ USG } & SG \\
\hline Simple & $\mathrm{Y}=0.010 \mathrm{x}+0.785$ & $\mathrm{Y}=0.008 \mathrm{x}+0.920$ \\
\hline City & $\mathrm{Y}=0.009 \mathrm{x}+1.115$ & $\mathrm{Y}=0.008 \mathrm{x}+0.689$ \\
\hline
\end{tabular}

Table 1. Regression model for groups and scene conditions. $Y=\ln$ (standardized dispersion), $\mathrm{X}=\mathrm{FOV}$
Brandt, Dichgans and Koenig [2] reported that only when the retinal periphery more eccentric than $30^{\circ}$ was stimulated, was circular vection induced. For our experiment in the simple scene condition, the most significant dispersion difference was for the $90^{\circ}-120$ FOV interval. This finding is consistent with the Brandt et al. report. However, the results from our experiment showed that even the $30^{\circ}$ FOV condition evoked substantial postural disturbance (1.2 times as large as the baseline condition for standardized dispersion and 1.7 times more than the baseline condition for standardized rating). This is different from the Brandt et al. findings. Warren and Kurtz [21] reviewed several experiments that contradicted Brandt's et al. peripheral dominance hypothesis - that peripheral vision is specialized for self-motion perception. Warren and Kurtz found that even FOVs as small as $10^{\circ}-25^{\circ}$ evoked self-motion perception. Our data are consistent with their findings.

Our study examined effects on balance. We have addressed balance for two reasons. First, Kennedy and Stanney [12] have evaluated postural stability measures for assessing aftereffects from virtual environment exposure. Several studies [3], [6] also indicate that balance disturbance correlates highly with SS; consequently, balance disturbance may be considered a surrogate for simulator sickness intensity. Secondly, it is much easier to recruit subjects for an experiment on balance performance, which has no aftereffects, than for one on motion sickness, which often has substantial aftereffects. FOV could be a critical factor for simulator sickness.

\section{Conclusion}

The results from this experiment indicated that postural stability varied as a function of display FOV when watching a moving scene. Subjects exhibited more balance disturbance with increasing FOV. Scene content may influence self-motion perception and postural stability. This implies that when we present scenes with different contents, different levels of interactivity, and different resolutions in immersive environments, different FOVs may be required to achieve a minimum level of 'presence'. Future research will focus on interactions among resolution, FOV and scene content.

\section{Acknowledgments}

Supported by NASA Grant NAG5-4074 and an Eastman Kodak Contract. We thank D. L. Harm for 
loaning us the balance platform used in this study and H. Abi-Rached for software development.

\section{REFERENCES}

[1] G.V. Barrett, C.L. Thornton, and P.A. Cabe, Cue conflict related to perceptual style. Journal of Applied Psychology, 54(3), 1968, pp258-264

[2] T. Brandt, J. Dichgans, and E. Koenig, Differential effects of central versus peripheral vision on egocentric and exocentric motion perception. Experimental Brain Research, 16, 1973, pp476-491

[3] S.V.G. Cobb and S.C. Nichols, Static posture tests for the assessment of postural instability after virtual environment use. Brain Research Bulletin, 47(5), 1998, pp459-464

[4] J. Dichgans, and T. Brandt, Visual-vestibular interaction: Effects on self-motion perception and postural control. In Held, R., \& Leibowitz, H.W. (Eds.), Handbook of sensory physiology (Vol. VIII). New York: Springer-Verlag, 1978

[5] P. DiZio, and J.R. Lackner, Circumventing side effects of immersive virtual environments. In Smith, M., \& Salvendy, R. (Eds), Design of computing system: Social and ergonomic considerations, Amsterdam: Elsevier, 1997, pp. 893-896.

[6] K.M. Hamilton, L. Kantor, and L.E. Magee, Limitations of postural equilibrium tests for examining simulator sickness. Aviation, Space, and Environment Medicine, 59, 1989, pp246-251

[7] R. Held, Two modes of processing spatially distributed visual stimulation. In Schmitt, F.O. (Ed.), The neurosciences: Second study program. NY: Rockefeller University Press, 1970

[8] R. Held, J. Dichgans, and J. Bauer, Characteristics of moving visual scenes influencing spatial orientation, Vision Research, 15, 1975, pp357-365

[9] P.J. Hezel, and H. Veron, Head-mounted displays for virtual reality. Society for Information Display International Symposium Digest of Technical Papers. Vol XXIV. Playa Del Ray, CA: society for information display. 1993, pp909-911

[10] I.P. Howard, and T. Heckmann, Circular vection as a function of the relative sizes, distances and positions of two competing visual displays. Perception, 18, 1989, pp657-667

[11] J. Hulk, and F. Rempt, Vertical optokinetic sensations by limited stimulation of the peripheral field of vision. Ophthalmologica, 186, 1983, pp97-103

[12] B. Isableu, T. Ohlmann, J. Cremieux, and B. Amblard, Selection of spatial frame of reference and postural control variability. Experiment Brain Research, 114, 1997, pp584-589

[13] R.S. Kennedy, and K.M. Stanney, Postural instability induce by virtual reality exposure: Development of certification protocol. International Journal of Human-computer Interaction, 8(1), 1996, pp25-47

[14] R.S. Kennedy, L.J. Hettinger, and M.G. Lilienthal, Simulator sickness. In Crampton, G.H. (Ed.), Motion and space sickness (pp317-341). Boca Ration, FL: CRC Press. 1990

[15] R.S. Kennedy, M.G, Lilienthal, K.S. Berbaum, D.R. Berbaum, and McCauley, M.E., Simulator sickness in U.S. Navy flight simulators. Aviation, Space and Environmental Medicine, 60(1), 1989, pp10-16

[16] R.V. Kenyon, and E.W. Kneller, The effects of filed of view size on the control of roll motion. IEEE Transactions on Systems, Man, and Cybernetics. 23(1), 1993, pp183-193

[17] Keshner, E. and Kenyon, R.V., The influence of an immersive virtual environment on the segmental organization of postural stabilizing responses, Journal of Vestibular Research, in press

[18] H.W. Leibowitz, and R. B. Post, The two modes of processing concept and some implications In Beck, J.(Ed.), Organization and representation in perception. Mahwah, NY: Erlbaum, 1982

[19] Parker, D.E., Duh, H.B.L, Philips, J.O., and Furness, T.A., Self-motion system frequency response: Implications for cybersickness. To appear in Proceedings of Second Biennial Space Biomedical Investigators Workshop, Galveston TX, Jan 2001

[20] J.D. Prothero, and H.D. Hoffman, Widening the field-of-view increase the sense of presence within immersive virtual environments (Human Interface 
Technology Laboratory Tech. Rep. R-95-4). Seattle: University of Washington, 1995

[21] W.H. Warren, and K.J. Kurtz, The role of central and peripheral vision in perceiving the direction of selfmotion. Perception and Psychophysics. 51(5), 1992, pp443-454

[22] L. Wolpert, Field-of-view information for selfmotion perception, In Warren, R. \& Wertheim, A. H.(Ed), Perception \& control of self-motion, NJ: LEA, 1990 